\title{
Analysis of signal to noise and directivity characteristic: of DAS VSP at near and far offsets - A CO2CRC Otway Project data example
}

\author{
Julia Correa ${ }^{1,2}$, Anton Egorov ${ }^{1,2}$, Konstantin Tertyshnikov ${ }^{1,2}$, Andrej Bona ${ }^{1,2}$, Roman Pevzner ${ }^{1,2}$, Tim Dean ${ }^{1}$, Barry Freifeld ${ }^{3}$, and Steve Marshall ${ }^{2}$
}

\begin{abstract}
During the last decade, distributed acoustic sensing (DAS) has emerged as a new technology for seismic acquisition. DAS has the potential to reduce the cost of permanent monitoring operations over time as it offers long equipment survivability and requires minimum maintenance. However, broad adoption of DAS technology still faces some challenges, such as low sensitivity and high levels of noise compared to conventional seismic sensors. Recent developments in fiber-optic systems and cable designs aim to overcome these limitations. To understand how DAS can be used in monitoring applications, it is important to know how it behaves with varying offsets and incidence angles. An offset VSP survey was acquired, at the CO2CRC Otway Project, using a straight single-mode fiber, a straight "enhancedbackscatter" fiber, and a conventional three-component geophone tool. The results from this survey show that DAS has the potential to provide similar, or even superior, quality data sets as conventional geophones.
\end{abstract}

\section{Introduction}

During the last decade, distributed acoustic sensing (DAS) has emerged as a new technology for seismic acquisition. DAS has the potential to acquire acoustic data at high spatial sampling along the length of a fiber-optic cable. DAS is particularly promising for permanent monitoring as the cable can be installed permanently, offers long-term equipment survivability, and requires minimum maintenance. Permanent monitoring can aid in increasing reservoir production and operations safety, though it is still an expensive practice. DAS has the potential to decrease significantly the cost of permanent monitoring operations over time.

DAS acquires acoustic signal along a single-mode fiber similar to those used in standard telecom cables. Differences in the phase of the backscattered light are attributed to strain on the cable caused by impinging acoustic waves. Broad adoption of DAS technology still faces some challenges, such as low sensitivity and high levels of noise compared to conventional acoustic sensors. Recent developments in fiber-optic systems and cable designs aim to overcome these issues.

To understand how DAS can be used in monitoring applications, it is important to know how it behaves with varying offsets and incidence angles. Here, we show data from the CO2CRC Otway Project. The project, located in Victoria, Australia, is the first Australian $\mathrm{CO}_{2}$ geosequestration demonstration project. Since its inception in 2006, the Otway Project site has been developed as a world-class research facility for $\mathrm{CO}_{2}$ geosequestration research through a series of test injections into various geologic formations. Seismic imaging of the evolution of the injected $\mathrm{CO}_{2}$ plumes has been carried out to provide assurance that the gas is safely stored in the subsurface and to validate our understanding of the behavior of $\mathrm{CO}_{2}$. Stage 1 of the project, completed in 2010, utilized conventional surface 4D seismic in conjunction with $4 \mathrm{D}$ vertical seismic profiling (VSP) acquired with geophones to conduct primarily assurance monitoring of $65,000 \mathrm{t}$ of a $\mathrm{CO}_{2} / \mathrm{CH}_{4}$ gas mixture injection into a depleted gas reservoir at $2 \mathrm{~km}$ depth (Jenkins et al., 2012; Gurevich et al., 2014). The Stage $2 \mathrm{C}$ study successfully imaged the evolution of a small (5000-15,000 t) plume at $1.5 \mathrm{~km}$ depth using a buried geophone array and 4D VSP (Pevzner et al., 2017). Starting from a successful trial of DAS conducted on the site in 2012 (Daley et al., 2013), this technology became an important component of the seismic monitoring program. The Otway installation includes both a buried 3D DAS array and a fiber-optic cable on production tubing in the CRC-2 well (Correa et al., 2017). Due to the exceptionally broad seismic data coverage available for the site, it offers an ideal environment for testing the performance of DAS as a monitoring tool.

The new phase of the Otway Project, Stage 3, aims to establish cost-effective solutions for monitoring injected $\mathrm{CO}_{2}$, focusing on multiwell VSP acquisitions. The first appraisal well for Stage 3 (CRC-3) was drilled in early 2017, with additional wells scheduled to be installed in 2018. The CRC-3 well was instrumented with two fiber-optic cables cemented behind the casing. The cables carry a combination of multimode fibers for temperature measurements and both standard single-mode fibers and a newly developed fiber with increased sensitivity. Here we show the results of an offset VSP survey acquired using both types of fiber (standard single-mode and increased sensitivity fibers) and a conventional three-component (3C) geophone tool. We aim to compare DAS response from both systems and understand how it behaves with varying offsets and incidence angles. We hope the results from this survey will assist decision making when designing monitoring surveys with DAS technology.

\section{Survey design and acquisition}

The VSP data acquisition was conducted in May 2017 to improve seismic characterization of the subsurface, appraise the capability of the fiber-optic system, estimate its performance in different survey geometry configurations, and compare it with a conventional seismic locking-arm geophone array. The survey was conducted in the CRC-3 well, the first injector well for Stage 3 of the Otway Project. CRC-3 was drilled in the first quarter of 2017 to a depth of $1667 \mathrm{~m}$. The completed CRC-3 
well is instrumented with several designs of fiber-optic cables, which are clamped along the long string of casing and cemented in place.

During the survey, the seismic signal was recorded with two different optical interrogators. The second-generation DAS interrogator developed by Silixa Ltd., referred to as an iDAS v2, was connected to the standard single-mode straight fiber. The latest version of Silixa's iDAS, which we refer to as iDAS v3, consists of an interrogator with optimized architecture for use with a proprietary optical fiber (Constellation), exhibiting engineered backscattering properties. The Constellation fiber was designed to allow more bright backscattered light to reach the interrogator with an optimal ratio between forward propagating and reflecting laser energy. Sercel's SlimWave downhole array consisting of eight shuttles with 3C geophones (Omni-2400 15 Hz) was deployed in the CRC-3 well and acquired data simultaneously with the fiber-optic systems.

Five locations for the offset VSP shot points, SP0, SP1, SP5, SP6, and SP7 (using continuous offset shot point numbering for all stages of the project; SP0 and SP1 were used in previous surveys), were chosen to cover a range of various azimuths and distances for the experiment (Figure 1). Shot points SP7 and SP0 are check shot positions for the CRC-3 and CRC-1 wells, respectively. Far offsets were mainly employed to explore potential limitations of DAS sensitivity at large distances.

The offset shot point locations were:

- $\quad$ shot point 7: $~ 50 \mathrm{~m}$ from $\mathrm{CRC}-3$ well

- shot point 0: $\sim 680 \mathrm{~m}$ from CRC-3 well

- shot point 1: $970 \mathrm{~m}$ from CRC-3 well

- $\quad$ shot point 5: 1025 $\mathrm{m}$ from CRC-3 well

- $\quad$ shot point 6: 2000 $\mathrm{m}$ from CRC-3 well

For every offset shot point, the geophones depth range in the well is $295-1600 \mathrm{~m}$, with a receiver spacing of $15 \mathrm{~m}$. Both DAS records acquired the entire length of the well $(0-1667 \mathrm{~m})$ with a $1 \mathrm{~m}$ recording spatial sampling.

A walkaway VSP (wVSP) survey was also acquired along existing roads, using 369 vibration points. The wVSP was conducted using 26,000 lb Inova UNIVIB vibrator trucks, with source parameters set to $6-150 \mathrm{~Hz}$ linear $24 \mathrm{~s}$ sweep with a $4 \mathrm{~s}$ listen time. In total, five sweeps were shot per vibration point at the offset locations.

\section{Conversion of DAS response to vertical particle velocity and comparison with geophone measurements}

We aim to quantify the difference in the data quality of DAS and geophone measurements. Since DAS and geophones measure different physical quantities, a conversion from one to the other is necessary for such a comparison. Herein, we convert DAS data to the particle velocity, which is measured by geophones within an appropriate frequency range. Moreover, converting DAS data to particle velocity allows one to apply existing processing and imaging routines to the converted DAS data. An example of such a technique is full-waveform inversion (Virieux and Operto, 2009; Egorov et al., 2017).

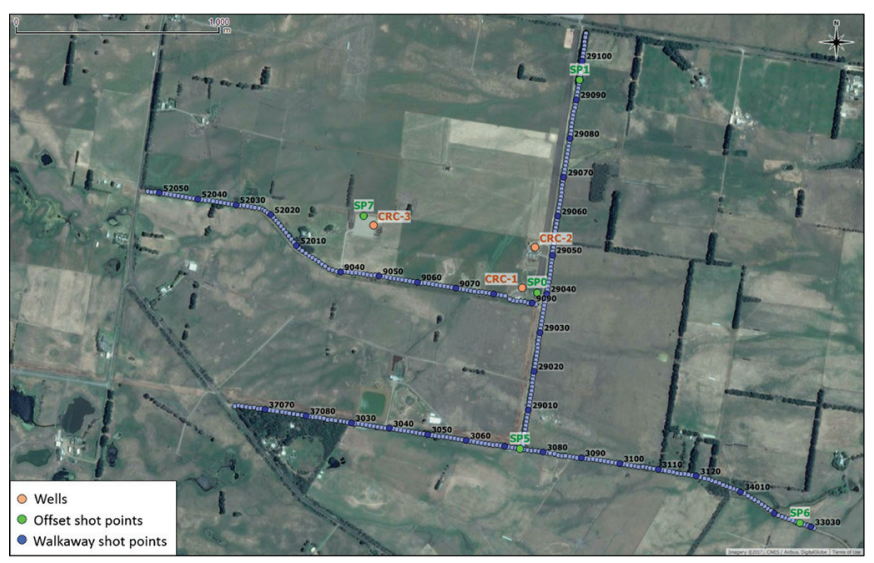

Figure 1. Survey map for the VSP acquisition in CRC-3 well, C02CRC Otway research site.

DAS measures strain, or its rate of change, in the optical fiber (Parker et al., 2014). DAS response can be approximated as (Bona et al., 2017)

$$
\frac{d}{d t} \int_{L}(u(z-G / 2+l, t)-u(z+G / 2+l, t)) w(l) d l,
$$

where $u$ is the displacement along the direction of the fiber, $G$ is the gauge length, $L$ is the pulse width, and $w(l)$ is the function that defines the laser pulse shape. Several techniques may be used for the conversion of DAS data to particle velocity. Time integration is one of the methods commonly used for that purpose. Daley et al. (2016) apply rescaling of the time-integrated data using the local propagation velocity of seismic waves for the comparison with geophones. This technique requires knowledge of seismic velocities and does not automatically account for the wave propagation direction. Another approach is to integrate the data along the cable length. Herein, we use the conversion derived from equation 1 , in which the displacement $u$ is given by a monochromatic plane wave $A e^{-i(\omega t-\vec{k} \vec{x})}$. The derived DAS response is similar to that used by Bakku (2015) and Dean et al. (2017), with the exception of including the pulse length:

$$
A_{z} e^{-i\left(\omega t-k_{z} z\right)} \frac{\omega}{k_{z}}\left(e^{i k_{z} L / 2}-e^{-i k_{z} L / 2}\right)\left(e^{i k_{z} G / 2}-e^{-i k_{z} G / 2}\right),
$$

which compares to the response of a geophone:

$$
-i \omega A_{z} e^{-i\left(\omega t-k_{z} z\right)} \text {. }
$$

Thus, the filter converting the DAS response to geophone response is equal to the ratio of expressions 2 and 3:

$$
\frac{i k_{z}}{4 \sin \left(k_{z} L / 2\right) \sin \left(k_{z} G / 2\right)}
$$


Filter 4 can be used to process the data directly. To avoid division by zero for certain values of $k_{z}$, the filter can be regularized by adding a small parameter $\gamma$ to the denominator. This parameter is similar to the water-level parameter commonly used in deconvolution. To pick $\gamma$, we first apply the filter without regularization, which correctly converts the waveforms but may contain noise in the output. Next, we test several $\gamma$ values, compare each of the results to the unregularized processing output, and pick the smallest $\gamma$ that attenuates the unwanted noise while preserving the waveforms of the unregularized result.

For small values of the wave vector component $k_{z}$, the filter 4 can be simplified by approximating $\sin \left(k_{z} L / 2\right) \approx k_{z} L / 2$ and $\sin \left(k_{z} G / 2\right) \approx k_{z} G / 2$ :

$$
-\frac{1}{i k_{z} L G}
$$

which is simply an integration along the fiber with reversed polarity multiplied by a scalar $1 / L G$. As the absolute value of seismic samples is usually given in arbitrary units, that scalar can be dropped.
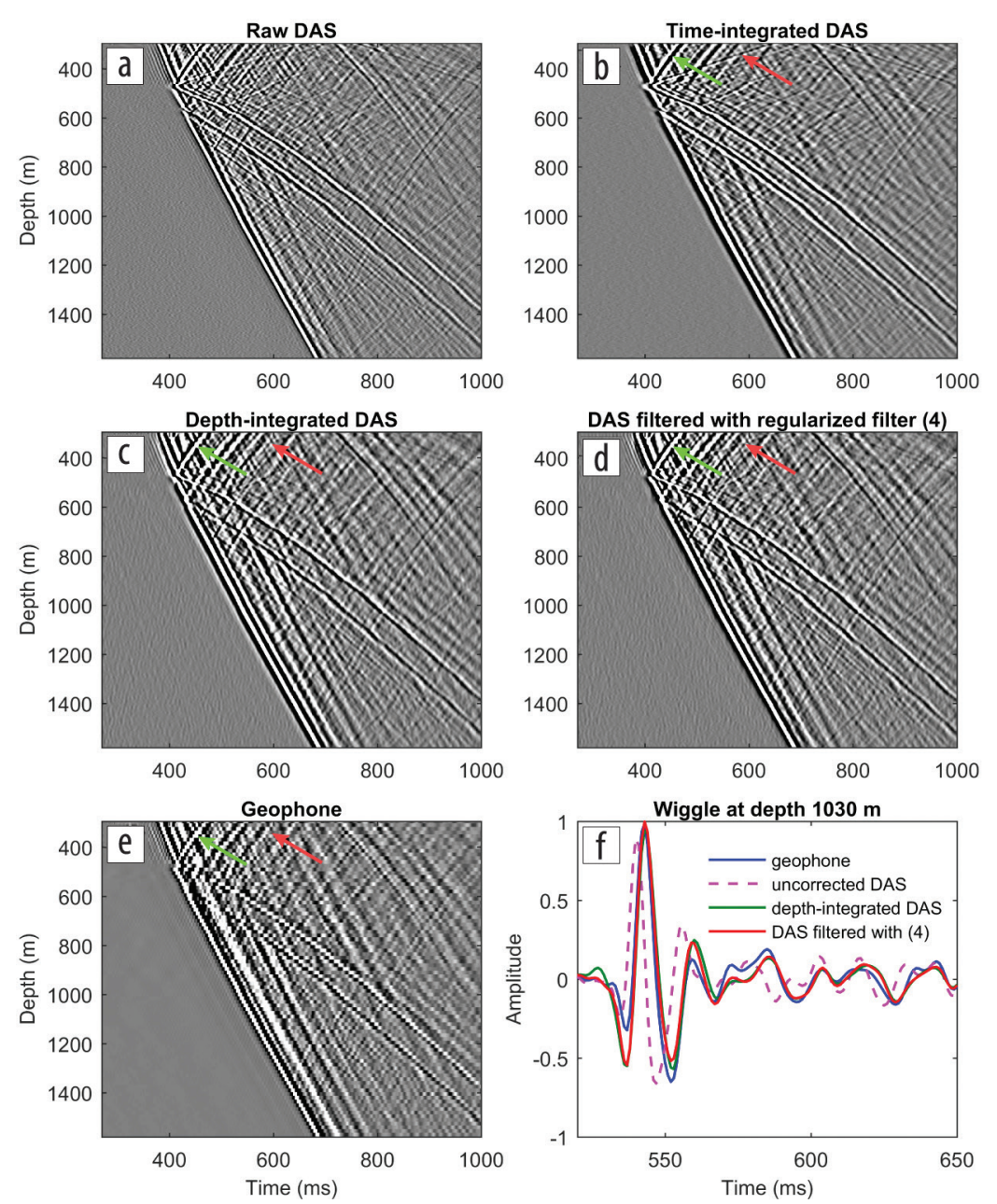

Figure 2. Conversion of DAS data to vertical component of particle velocity. (a) Raw DAS gather, (b) DAS gather after time integration, (c) DAS gather after depth integration, (d) DAS gather after correction using regularized filter, (e) geophone gather, and (f) comparison of traces at depth of $1030 \mathrm{~m}$. Green and red arrows show differences between the conversion results.
The presented theory provides an easy way of correcting for both gauge length and pulse width. Dean et al. (2017) show that the effect of pulse width is significantly more subtle when compared to the effect of gauge length for pulse width smaller than approximately half the gauge length. The effect of pulse width is mostly evident for wavenumbers larger than the first zero of $\sin \left(\frac{k G}{2}\right)$.

To compare the results of the different types of conversion procedures, we use the data for shot point SP0, with offset of approximately $680 \mathrm{~m}$. Figure 2a shows a raw DAS gather. This gather is integrated along the time axis (Figure 2b) and along the depth axis (Figure 2c). After the integration, we use median filter in the $(x, t)$ domain to filter out low-wavenumber artifacts caused by the numerical integration. Figure $2 \mathrm{~d}$ shows the gather corrected using the regularized filter 4 with the regularization coefficient $\gamma=0.0008$. A reference gather acquired with geophones in the same well is shown in Figure 4e where the coarse depth sampling is clearly visible.

It is clear from the comparison of time-integrated DAS and geophone gathers that time integration does not correct the polarity of upgoing waves (the upgoing wavefields in Figure 2b and Figures 2c-e have opposite signs, which is indicated by green arrows). Wavefields in Figures $2 \mathrm{c}-\mathrm{d}$ show that the depth integration and the regularized filter 4 correct both the wavelet shape and the sign of the upgoing wavefield. Another difference between the conversion results is that the time-integrated result contains a visible converted reflected wave from a layer at $\sim 500 \mathrm{~m}$ depth (indicated by red arrows). This wave has very low amplitude on other conversion results and the geophone gather.

We pick a single trace at a depth of $1030 \mathrm{~m}$ from the gathers and compare these traces in Figure 2f. This comparison shows that, for the displayed gather, the depth integration and the regularized filter produce similar results. However, even for these filters, there is still a difference between the converted DAS traces and the geophone trace.

Comparison of the wavefield acquired from both DAS systems and geophones shows that for the nearest offset SP7 (Figures $3 \mathrm{a}-\mathrm{c}$ ), the three acquired data sets look similar. The geophones are able to clearly record $\mathrm{P}$-wave reflections along the well, as expected. The level of noise on the data is low, except for the presence of the side lobes resulting from crosscorrelation with the vibroseis sweep, which are present in all three data sets. Geophone data are 
affected by tube wave noise as the tool is suspended in the well. On DAS gathers, the tube wave is almost absent (indicated by green arrows). DAS v3 shows a defect in the fiber at $1400 \mathrm{~m}$, which occurred during the optical fiber fabrication process.

For the VSP acquired at a medium offset at shot point SP0, at approximately $600 \mathrm{~m}$ distance (Figures 3d-f), differences between geophones and DAS wavefields become more obvious due to differences in directional sensitivities of the receivers. For this shot point, both DAS systems are able to acquire the same $\mathrm{PP}$-wave reflections as acquired by the geophones. The main difference between the DAS systems and geophones at this offset is seen at approximately $500 \mathrm{~m}$ depth, where DAS also acquires PS-wave reflections.

At shot point SP5 (Figures 3g-i) and SP6 (Figures 3j-1), at approximately 1 and $2 \mathrm{~km}$ distance, DAS v3 and v2 still compare reasonably well to geophone data. DAS is able to record upgoing and downgoing waves, as well as PS-waves. DAS v2 shows a significantly higher level of random noise when compared to DAS v3. Some events that arrive almost parallel to the fiber (indicated by red arrows) were not reconstructed by the conversion.

\section{Comparison of signal-to-noise ratios}

To analyze the performance across all recording systems for different offsets, we compare the VSP records acquired using geophones iDAS v2 with standard fiber and iDAS v3 with enhanced fiber at shot points SP7, SP0, SP5, and SP6 (shown in
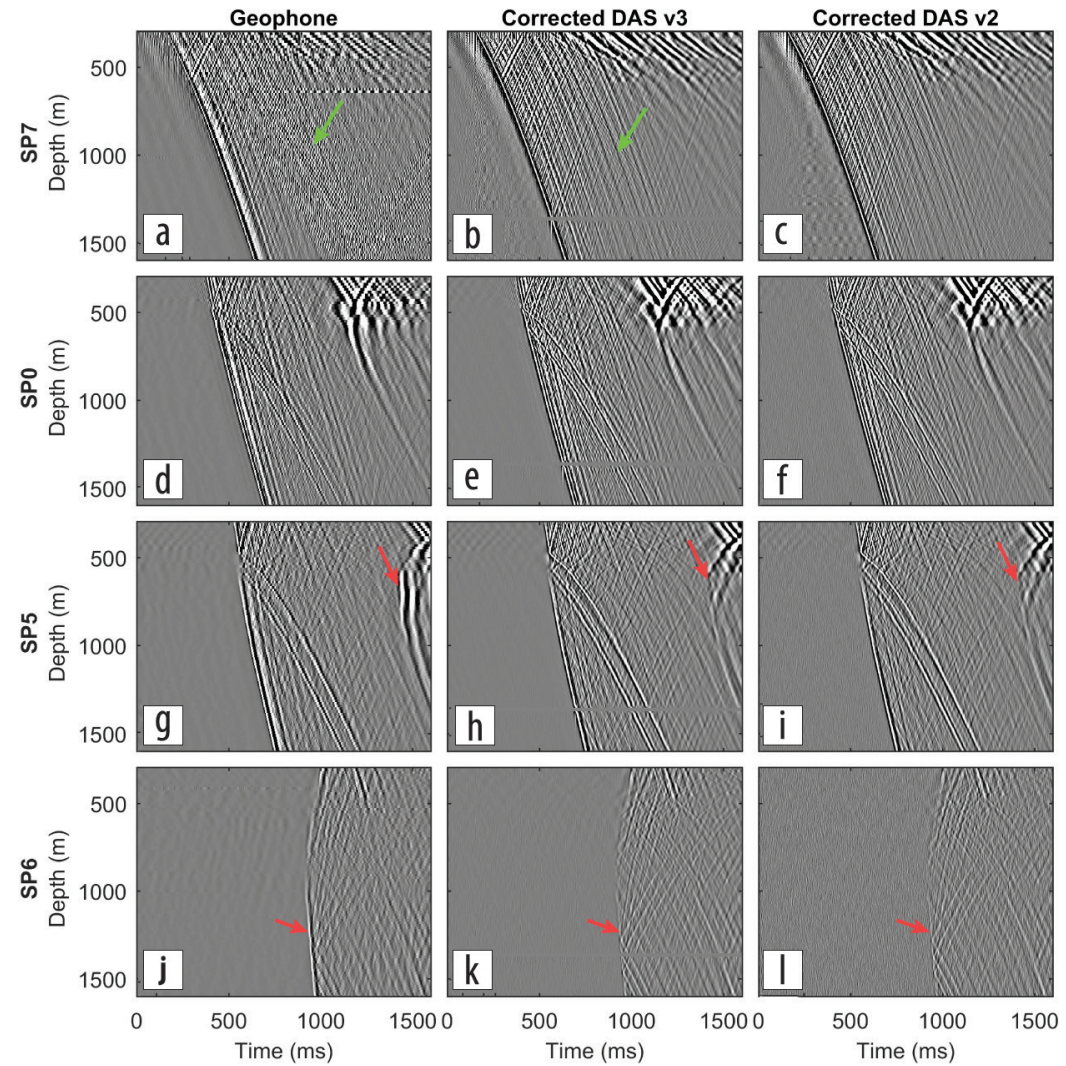

Figure 3. (a, d, g, and j) Offset points SP7, SP0, SP5, and SP6 for geophone data, (b, e, h, and k) converted DAS v3, and $(\mathrm{c}, \mathrm{f}, \mathrm{i}$, and $\mathrm{I})$ converted DAS v2. Green and red arrows show differences between DAS gathers and geophone gather. Overall, DAS systems are able to record the same PP events as recorded by the geophones.
Figure 4) that represent near to far offsets. Since DAS senses the strain changes only along the direction of the fiber, we utilize only the vertical component of the geophones for the comparison. Unlike the geophones, DAS acquires the data along the entire length of the well simultaneously. By the end of the geophone acquisition, DAS acquired more than 50 shots for each offset point. To increase the signal-to-noise ratio $(\mathrm{S} / \mathrm{N})$, we stack all the repeated shots for DAS data at each location. While the transformation of DAS measurements to the vertical particle velocity is important for comparison of the waveforms recorded by the different systems, for comparison of the $\mathrm{S} / \mathrm{N}$ of the systems we opt to use the strain response of DAS, which we obtain by integration of the strain rate along time. We choose to use strain not only due to its wide use in the industry but also because the time integration does not contain artifacts that can be produced by the conversion to the particle velocity by the zeros in the presented filter. It is important to note that different conversions of the data might result in different $\mathrm{S} / \mathrm{N}$ values.

$\mathrm{S} / \mathrm{N}$ values were calculated by dividing the root-mean-square (rms) amplitude of a 20 ms window around the first breaks by the mean rms amplitude of a $20 \mathrm{~ms}$ window of noise in the beginning of the record, from depth 1000-1200 m. This interval was chosen as it gives a good representation of the background noise, free of correlation side lobe effects. Due to the high amplitude of the first breaks in comparison with reflected waves, this method of $\mathrm{S} / \mathrm{N}$ estimation could, though, yield optimistic results that do not represent the $\mathrm{S} / \mathrm{N}$ of reflected waves.

Figure 4 shows that the $\mathrm{S} / \mathrm{N}$ of geophones, DAS v2, and DAS v3 decreases with distance, as expected. Though, S/N in DAS v2 seems to decrease at a greater rate than geophones when acquiring at further offsets geophones decay by $10 \mathrm{~dB}$, while DAS v2 decay by $20 \mathrm{~dB}$ (Figures $4 \mathrm{~d}$ and 41). DAS v3 performance is significantly better than DAS v2 as $\mathrm{S} / \mathrm{N}$ virtually remains the same. Visually, all systems present good quality records, showing similar reflections.

The $\mathrm{S} / \mathrm{N}$ of the geophone data at a distance of $50 \mathrm{~m}, \mathrm{SP} 7$, is approximately $10 \mathrm{~dB}$ higher than for the DAS data sets. For this distance, DAS v2 and v3 data sets have no significant difference in $\mathrm{S} / \mathrm{N}$ (Figure 4d). While distance increases to $680 \mathrm{~m}$ at $\mathrm{SP} 0$, the $\mathrm{S} / \mathrm{N}$ of DAS v2 decreases significantly, showing a decay of approximately $10 \mathrm{~dB}$. DAS v3 shows similar $\mathrm{S} / \mathrm{N}$ in comparison to geophone data (Figure 4h). The apparent $\mathrm{S} / \mathrm{N}$ discrepancy between DAS and geophones for the near offset can be attributed to the presence of noise likely caused by the air blast from the source (seen at approximately $200 \mathrm{~ms}$ ). 
At shot point SP5 (Figure 41), $1025 \mathrm{~m}$ from the well, DAS v3 and geophone data sets still have similar quality, while DAS v2 suffers a further $10 \mathrm{~dB}$ loss. When data is acquired at $\mathrm{SP} 6$, at a $2 \mathrm{~km}$ distance, geophones $\mathrm{S} / \mathrm{N}$ further decreases by $10 \mathrm{~dB}$ (Figure $4 \mathrm{p}$ ). Because of the directivity, first breaks are not present in the DAS data at offset $\mathrm{SP} 6$, therefore $\mathrm{S} / \mathrm{N}$ was not calculated. Upgoing and downgoing reflections are well imaged in both DAS v3 and DAS v2, despite the high level of random noise present in the latter. Directivity will affect waves closer to normal incidence, such as direct arrivals. However, DAS is still sensitive to reflected waves; they are better captured on DAS data due to the higher spatial sampling.

To increase $\mathrm{S} / \mathrm{N}$, DAS data often can be stacked with repeated sweeps so they constructively add in signal and decrease random noise. $\mathrm{S} / \mathrm{N}$ is calculated for DAS records after stacking of five sweeps and approximately 50 sweeps (maximum number of sweeps for each shot point varies from 48 to 60 ). Figure 5 shows a histogram of the difference in $\mathrm{S} / \mathrm{N}$ between five and 50 stacked shots. The difference represents the improvement after stacking. For both DAS systems, $\mathrm{S} / \mathrm{N}$ increases by approximately $10 \mathrm{~dB}$. This shows that the improvement in $\mathrm{S} / \mathrm{N}$ varies close to $\sqrt{ } N, N$ being the number of stacks. This suggests that the noise on DAS data is predominantly random. For far offsets on DAS v3, however, the improvement is slightly inferior, presenting approximately $7 \mathrm{~dB}$ of increase in $\mathrm{S} / \mathrm{N}$.

\section{Directional sensitivity of DAS measurements}

Direction of particle displacement affects the sensitivity of DAS systems drastically. Figure 6 illustrates the decay of first break amplitudes observed in DAS v3 and v2. To highlight the variations in amplitude caused by directivity, it is useful to eliminate the effects of the spherical divergence and attenuation. This is done by normalizing the DAS amplitudes by the magnitudes of geophone $3 \mathrm{C}$ measurements.

At depths from 295 to $1600 \mathrm{~m}$, incidence angles for shot point $\mathrm{SP} 7$ vary from $10^{\circ}$ to $2^{\circ}$. At offset $\mathrm{SP} 0$, angles vary from $67^{\circ}$ to $23^{\circ}$ and at offset SP5, from $73^{\circ}$ to $31^{\circ}$. Amplitudes for both DAS systems decay approximately as cosine square of the angles of incidence, as predicted by Kuvshinov (2016) for straight singlemode fibers. The scatter in the normalized amplitudes is likely caused by the interference of the direct and reflected waves and noise. For small incidence angles, amplitudes show an unexpected trend, probably caused by the normalization with geophone amplitudes, which are influenced by the compaction of the ground after repeated sweeping at the same location. While geophones

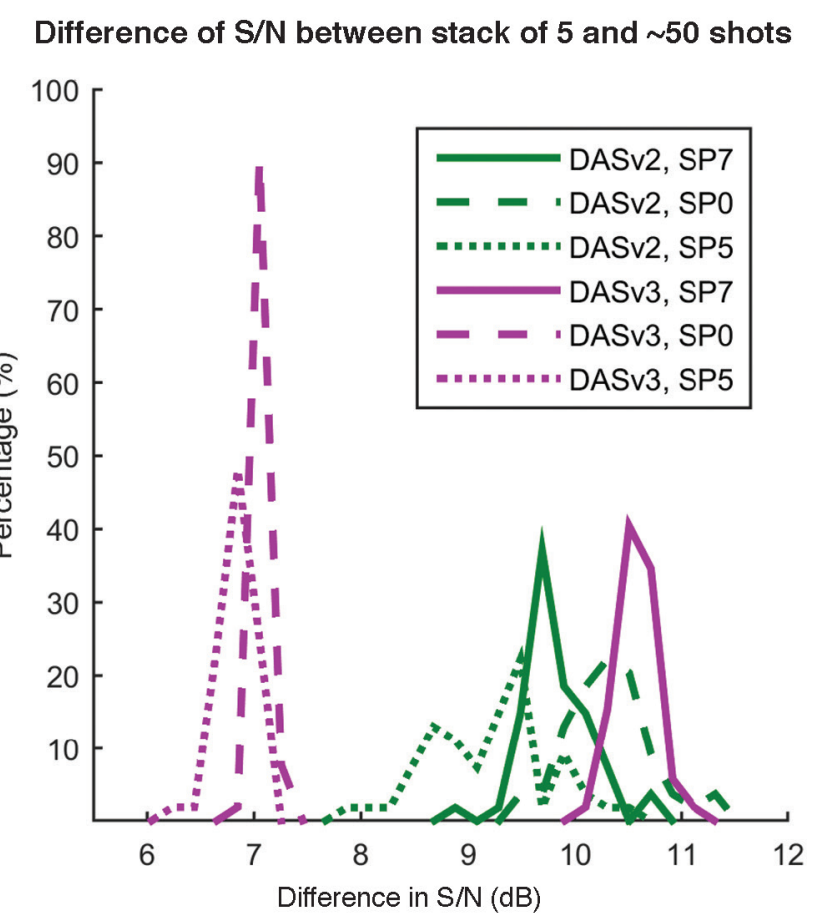

Figure 5. Difference in $S / N$ between DAS data stacked with five sweeps and approximately 50 sweeps. Differences in S/N for DAS v2 are in green, and those for DAS v3 are in purple. Overall, the improvement in $S / N$ of data sets is close to $10 \mathrm{~dB}$, which corresponds to the square root of number of stacks. 
acquire the data at each section of the well at a time, its amplitudes present a slight change in source signature along the well.

\section{Discussion and conclusions}

DAS offers many advantages over the use of conventional geophones. However, it is often affected by the lack of sensitivity and high noise levels inherent in DAS data. Furthermore, straight fiber has a narrower directivity pattern compared to geophones. While these factors still hinder the broader application of DAS in the seismic industry, DAS technology continues to evolve rapidly. Thus, it is important to reevaluate its capabilities in comparison to standard geophones. Understanding how DAS behaves in respect to varying offsets and how directivity influences its amplitudes is crucial for a successful adoption of DAS in permanent monitoring.

We show the results of a field trial at the $\mathrm{CO} 2 \mathrm{CRC}$ Otway site where we acquired VSP data using both $3 \mathrm{C}$ geophones and DAS cables cemented behind the casing with offset to shot point positions varying from $50 \mathrm{~m}$ to $1.8 \mathrm{~km}$. DAS was acquired using a standard single-mode fiber and a new type of fiber with enhanced backscatter.

For near offsets, both DAS systems show similar S/N, with geophones being approximately $12 \mathrm{~dB}$ higher. This comes with the same source effort used for DAS and geophone acquisition (same number of shots required for the full geophone VSP by moving the seismic string were used to stack DAS data). If we decrease the number of shots on DAS to mimic the number of shots recorded by geophones at each level, the difference would be greater. However, S/N on DAS data is high, and the record is visually similar to the geophone record. An important aspect is that, due to the higher spatial sampling, DAS data might provide more detailed velocity information compared to geophones.

$\mathrm{S} / \mathrm{N}$ for the single-mode fiber decreases significantly at far offsets, where it shows a loss of $20 \mathrm{~dB}$ at $1025 \mathrm{~m}$ distance compared

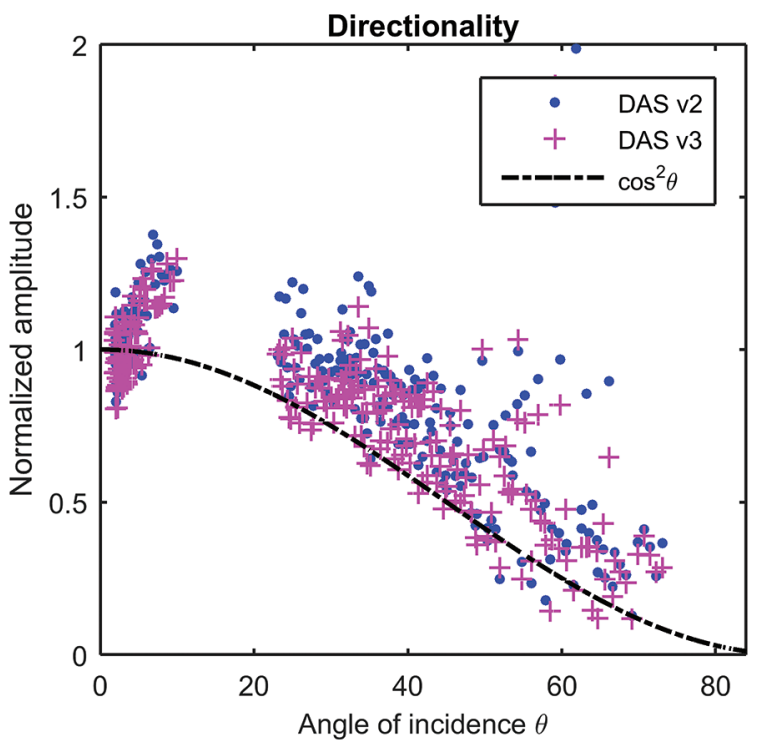

Figure 6. Directivity plot showing normalized amplitudes of DAS v3 and DAS v2 against angles of incidence. Theoretical cosine squared curve is plotted with the dashed line. to Constellation, where it remains high at far offsets. Although noise in single-mode fiber was significantly higher, reflected waves are well pronounced on all DAS records. There is no doubt that the DAS data can be used for imaging and monitoring purposes. It is also obvious that for the very far offsets (SP6, $1.8 \mathrm{~km}$ ) reflected waves are better captured by DAS compared to geophones due to higher spatial sampling.

In conclusion, from our perspective, data acquired with fiberoptic sensors deployed behind the casing potentially outperform geophone data for seismic monitoring applications. This is largely valid for both standard single-mode and Constellation fibers, but the latter fibers clearly provide higher quality data for the large offsets/incidence angles. However, in many cases, deployment of the fiber-optic cable behind the casing is not viable (for instance, when the well is already drilled or when there are risks of compromising a newly drilled well's integrity by deploying equipment behind the casing). We believe these are the cases where impact of such fiber with higher sensitivity will be the greatest.

To establish an accurate comparison, we have proposed a filter that converts DAS data to geophone equivalent. After conversion, the DAS signature becomes very close to the geophone signature, correcting for polarity differences of the upgoing and downgoing fields. Such conversion is also necessary when using DAS in applications that require particle velocity response, such as in full-waveform inversion. TIL:

\section{Acknowledgments}

The Otway Project received $\mathrm{CO} 2 \mathrm{CRC}$ funding through its industry members and research partners, the Australian Government under the CCS Flagships Programme, the Victorian State Government, and the Global CCS Institute. The authors wish to acknowledge financial assistance provided through Australian National Low Emissions Coal Research and Development supported by the Australian Coal Association Low Emissions Technology Limited and the Australian Government through the Clean Energy Initiative. We are grateful to Andy Clarke and Tom Parker (Silixa) for the advice on well instrumentation and DAS data acquisition. We also thank our colleagues from CO2CRC, LBNL, and Curtin University, in particular Professor Boris Gurevich, for their valuable support and contribution to this work.

Corresponding author: julia.correa@postgrad.curtin.edu.au

\section{References}

Bakku, S. K., 2015, Fracture characterization from seismic measurements in a borehole: PhD thesis, Massachusetts Institute of Technology.

Bona, A., T. Dean, J. Correa, R. Pevzner, K. Tertyshnikov, and L. Van Zaanen, 2017, Amplitude and phase response of DAS receivers: $79^{\text {th }}$ Conference and Exhibition, EAGE, Extended Abstracts, https://doi.org/10.3997/2214-4609.201701200.

Correa, J. C., B. M. Freifeld, M. Robertson, R. Pevzner, S. Bona, D. Popik, S. Yavuz, et al., 2017, Distributed acoustic sensing applied to 4D seismic - Preliminary results from the CO2CRC Otway site field trials: $79^{\text {th }}$ Conference and Exhibition, EAGE, Extended Abstracts, https://doi.org/10.3997/2214-4609.201700811. 
Daley, T. M., B. M. Freifeld, J. Ajo-Franklin, S. Dou, R. Pevzner, V. Shulakova, S. Kashikar, et al., 2013, Field testing of fiber-optic distributed acoustic sensing (DAS) for subsurface seismic monitoring: The Leading Edge, 32, no. 6, 699-706, https://doi. org/10.1190/tle32060699.1.

Daley, T. M., D. E. Miller, K. Dodds, P. Cook, and B. M. Freifeld, 2016, Field testing of modular borehole monitoring with simultaneous distributed acoustic sensing and geophone vertical seismic profiles at Citronelle, Alabama: Geophysical Prospecting, 64, no. 5, 1318-1334, https://doi.org/10.1111/1365-2478.12324.

Dean, T., T. Cuny, and A. H. Hartog, 2017, The effect of gauge length on axially incident $\mathrm{P}$-waves measured using fibre optic distributed vibration sensing: Geophysical Prospecting, 65, no. 1, 184-193, https://doi.org/10.1111/1365-2478.12419.

Egorov, A., R. Pevzner, A. Bona, S. Glubokovskikh, V. Puzyrev, K. Tertyshnikov, and B. Gurevich, 2017, Time-lapse full waveform inversion of vertical seismic profile data: Workflow and application to the CO2CRC Otway Project: Geophysical Research Letters, 44, no. 14, 7211-7218, https://doi.org/10.1002/2017GL074122.

Gurevich, B., R. Pevzner, M. Urosevic, A. Kepic, V. Shulakova, and E. Caspari, 2014, 2D and 3D seismic investigations for Stage 1 and $2 \mathrm{C}$, in P. J. Cook, ed., Geologically storing carbon: Learning from the Otway Project experience: CSIRO Publishing, 155-196.

Jenkins, C. R., P. J. Cook, J. Ennis-King, J. Undershultz, C. Boreham, T. Dance, P. de Caritat, et al., 2012, Safe storage and effective monitoring of $\mathrm{CO}_{2}$ in depleted gas fields: Proceedings of the National Academy of Sciences of the United States of America, 109, no. 2, E35-E41, https://doi.org/10.1073/pnas.1107255108.

Kuvshinov, B. N., 2016, Interaction of helically wound fibre-optic cables with plane seismic waves: Geophysical Prospecting, 64, no. 3, 671-688, https://doi.org/10.1111/1365-2478.12303.

Parker, T., S. Shatalin, and M. Farhadiroushan, 2014, Distributed acoustic sensing - A new tool for seismic applications: First Break, 32, no. 2, 61-69, https://doi.org/10.3997/1365-2397.2013034.

Pevzner, R., M. Urosevic, D. Popik, V. Shulakova, K. Tertyshnikov, E. Caspari, J. Correa, et al., 2017, 4D surface seismic tracks small supercritical $\mathrm{CO}_{2}$ injection into the subsurface: $\mathrm{CO} 2 \mathrm{CRC}$ Otway Project: International Journal of Greenhouse Gas Control, 63, 150-157, https://doi.org/10.1016/j.ijggc.2017.05.008.

Virieux, J., and S. Operto, 2009, An overview of full-waveform inversion in exploration geophysics: Geophysics, 74, no. 6, WCC1WCC26, https://doi.org/10.1190/1.3238367. 